(Julio-diciembre 2018)

Año 11. Académica sin Frontera

Núm. 28

ISSN: 2007-8870

\title{
http://revistainvestigacionacademicasinfrontera.com
}

\section{PRINCIPALES PROBLEMAS DE LA PYMES POR FALTA DE INFORMACIÓN OPORTUNA GENERADA EN SUS SISTEMAS CONTABLES}

\author{
PATRICIA Aguilar TAlamante ${ }^{1}$ \\ Universidad de Sonora, Unidad Centro \\ Alma BRENDA LEYVA CARRERAS ${ }^{2}$ \\ Universidad de Sonora, Unidad Centro \\ JosÉ ALFREDO HEREDIA BUSTAMANTE ${ }^{3}$ \\ Universidad de Sonora, Unidad Centro \\ MARÍA CANDELARIA GONZÁLEZ GONZÁLEZ ${ }^{4}$ \\ Universidad de Sonora, Unidad Centro
}

\section{Resumen}

La información que generan los Sistemas Contables se manifiesta como un factor de incidencia para las organizaciones en la concepción y persistencia de ventajas competitivas. Sin embargo, en últimas fechas se ha vuelto indispensable que la información esté bien administrada y sea de buena calidad. En esta investigación, se tomó como referencia a las Pymes afiliados a CANACINTRA en la ciudad de Hermosillo, Sonora y registrados en el SIEM con una fuerza de trabajo entre 10 y 250 trabajadores, una antigüedad mínima de cinco años en la actividad. El objetivo de la presente investigación es determinar con base en las opiniones de los empresarios Pymes, el grado de influencia que tienen el uso y la calidad

\footnotetext{
${ }^{1}$ Departamento de Contabilidad, Universidad de Sonora, Blvd. Luis Encinas y Rosales s/n, Colonia Centro, Hermosillo, Sonora Tel.01(642)2592211 y 12, correo electrónico: patricia.aguilar@unison.mx

${ }^{2}$ Departamento de Administración, Universidad de Sonora, Blvd. Luis Encinas y Rosales s/n, Colonia Centro, Hermosillo, Sonora, México Tel.01(662)259.22.11 correo electrónico: alma.leyva@unison.mx

${ }^{3}$ Departamento de Contabilidad, Universidad de Sonora, Blvd. Luis Encinas y Rosales s/n, Colonia Centro, Hermosillo, Sonora, México Tel.01(662)259.2168 correo electrónico: aheredia@pitic.uson.mx

${ }^{4}$ Departamento de Administración, Universidad de Sonora, Blvd. Luis Encinas y Rosales $s / n$, Colonia Centro, Hermosillo, Sonora, México Tel.01(662)259.2168 correo electrónico: mariacandelaria.gonzalez@unison.mx
} 
de la información generada por los Sistemas Contable para lograr ventaja competitiva. Los principales resultados muestran que para el empresario Pyme la información que genera los sistemas contables tiene influencia en las estrategias empresariales de su negocio y la calidad de la información tiene un impacto positivo para la toma de decisiones encaminada a la creación de estrategias empresariales que les ayude a lograr ventaja competitiva.

Palabras Clave: Sistema de información contable, Calidad de la información, Ventaja Competitiva.

\begin{abstract}
The information generated by the Accounting Systems is manifested as a factor of incidence for the organizations in the conception and persistence of competitive advantages. However, in recent dates it has become essential that the information is well managed and of good quality. In this research, reference was made to SMEs affiliated with CANACINTRA in the city of Hermosillo, Sonora and registered in the SIEM with a workforce of between 10 and 250 workers, a minimum of five years in the activity. The objective of this research is to determine, based on the opinions of SME entrepreneurs, the degree of influence that the use and quality of the information generated by Accounting Systems have in order to achieve a competitive advantage. The main results show that for the SME entrepreneur the information generated by the accounting systems has influence on the business strategies of their business and the quality of the information has a positive impact for the decision making aimed at the creation of business strategies that help them to achieve competitive advantage.
\end{abstract}

Key Words: Accounting information system, Information quality, Competitive Advantage.

\title{
Introducción
}

Desde el punto de vista gerencial, la Pequeña y mediana empresa (Pyme) presenta un marcado número de deficiencias entre las que se puede mencionar el ignorar aspectos de gestión como la calidad, la eficiencia, la competencia y el uso acertado de herramientas fundamentales. Una de las problemáticas de gestión de la Pyme, se presenta en el poco interés que tiene en dar un uso adecuado a las herramientas de control empresarial, entre las que se destaca el Sistema de Control Contable, y que, indudablemente, pueden contribuir a obtener información oportuna para mejorar la gestión empresarial. La poca valoración asignada a este tipo de herramientas ha significado un obstáculo en la rapidez y precisión de decisiones trascendentales.

Es reconocido que gran parte del sistema de información formal en las empresas está sustentado por la contabilidad, y de la eficacia de este sistema depende la rapidez y el acierto de los gerentes en sus decisiones. Referirse a las actividades y a los procedimientos formales destinados a tratar 


\section{http://revistainvestigacionacademicasinfrontera.com}

información relevante para la gerencia, es hablar de sistemas de información y, por ende, de su medio principal: la contabilidad.

La contabilidad deriva información indispensable para la administración y el desarrollo del sistema económico por ello, las empresas y en especial en las Pymes, recae la necesidad de implementar sistemas de información contable de tal forma que sea mucho más fácil la toma de decisiones para administradores, propietarios, inversionistas, proveedores, entre otros (García et al., 2006). Como consecuencia de lo anterior, la teoría de la contabilidad ha evidenciado una doble relación entre lo administrativo y lo contable, que corresponde a momentos históricos distintos de la disciplina. Un primer estado demuestra que lo administrativo determina o influencia lo contable, mientras que uno posterior ha evidenciado que lo contable ayuda a impulsar a las organizaciones (Hernández, 2006, pp. 206-208). De allí que la contabilidad y los costos se convierten en el elemento básico de las finanzas de una empresa, pues, mediante su adecuada utilización, puede realizarse una gestión eficiente de los activos de la empresa, representados por su valor económico (Vaivio y Sirén, 2010).

Por todo lo anterior, la contabilidad constituye una herramienta de control que funciona como el principal sistema de información que refleja el resultado de la toma de decisiones de los gerentes, así como un excelente banco de datos para predecir el futuro de las organizaciones. En consecuencia, la calidad en los procesos de información, y más los de carácter contable, determinan niveles de competitividad en las empresas, pues muchas de las decisiones tomadas son con base en los resultados o productos de dichos procesos. Partiendo de lo antes expuesto, el objetivo de la presente investigación es determinar con base en las opiniones de los empresarios Pymes, el grado de influencia que tienen el uso y la calidad de la información generada por los Sistemas Contable para lograr ventaja competitiva. 


\section{Revisión de literatura}

Desde un punto de vista genérico, la contabilidad en las organizaciones constituye una herramienta de suma importancia para que los directivos puedan, por una parte, administrar cada una de las actividades o áreas funcionales de las que son responsables, y, por la otra, coordinar las actividades o funciones dentro de la estructura organizativa como un todo (Horngren et. al., 2002, Vaivio y Sirén, 2010). Por ende, la contabilidad subyace como un valioso dispositivo de la normalización de los resultados, uno de los mecanismos de control con que cuentan las organizaciones para coordinar su trabajo y obtener información oportuna para la toma de decisiones.

Es reconocido que gran parte del sistema de información formal dentro de las empresas está sustentado por la contabilidad, y de la eficacia de este sistema depende la rapidez y el acierto de los gerentes en sus decisiones. Referirse a las actividades y a los procedimientos formales destinados a tratar información relevante para la gerencia, es hablar de sistemas de información y, por ende, de su medio principal: la contabilidad (Schekaiban y Ripoll, 2005). La contabilidad deriva información indispensable para la administración y el desarrollo del sistema económico. Esta información es proporcionada a quienes toman decisiones, como administradores, propietarios, inversionistas, proveedores, entre otros (Valvio y Sirén, 2010).

Desde el inicio de las operaciones de cualquier empresa, el sistema contable que se plantea utilizar debe corresponder con el aprovechamiento de los recursos disponibles y con los requerimientos de información por parte de la administración y de usuarios externos. Al respecto, las Pymes necesitan de un sistema contable flexible y sencillo, de tal forma que la gerencia pueda comprender su manejo y aprovechar la información que genera a los efectos de tomar decisiones acertadas, confiables y oportunas, reduciendo la incertidumbre a niveles mínimos (Peña, 2011). La misma autoría menciona que es pertinente tener en cuenta que una administración exitosa debe apoyarse en herramientas esenciales entre las que se cuenta un eficaz sistema de información contable; no sólo para dar cumplimiento a normativas tributarias sino también para 


\section{http://revistainvestigacionacademicasinfrontera.com}

proveerse de tecnologías de seguimiento y control de las transacciones y de las mismas decisiones.

La interrelación entre las variables para la competitividad y el aporte de los sistemas de información contable, se establece mediante los componentes que el desarrollo de los sistemas de información contable y su pertinencia con las necesidades organizacionales deben contemplar (Rueda y Arias, 2009). Significa lo anterior que parte de lo que caracteriza a la información contable -desde el punto de vista del mensaje financiero, de gestión y gerencial, y las prácticas de control entre otras- proviene de "presiones", desarrollos, cambios, concepciones, etc., desde la administración, entendida ésta como la gestión y distribución de recursos para la obtención de resultados de diverso orden (Munch, 2006).

El efecto de la capacidad de procesamiento de información sobre los recursos y competencias para realizar actividades y procesos empresariales transforma la manera como las organizaciones construyen sus relaciones con los demás agentes en su red de valor (Johnson, Scholes y Whittington, 2007). Cada organización es única (Hitt, Hoskisson y Ireland, 2001), en la medida en que posee diferentes recursos (físicos, humanos y capital organizacional) y capacidades, lo cual implica la obtención y uso diferenciados de información para sus propios propósitos. La información empresarial (incluyendo la contable) no sólo se genera para el cumplimiento de marcos normativos, sino para provecho de la organización (Rueda y Arias, 2009).

Los mismos autores mencionan que la información de los sistemas contables son el conjunto de mecanismos y herramientas utilizadas por las Pymes mediante los cuales obtiene información para mantener el control de los recursos y las actividades que realiza y, ofrece como ventaja ayudar a cumplir de manera puntual y constante con las obligaciones fiscales y contables de la entidad, además de ser de particular importancia para aquellas empresas con planes de expansión y crecimiento. Por su parte, Valvio y Sirén (2010), mencionan que de allí que la contabilidad se 
convierte en el elemento básico de las finanzas de una empresa, pues, mediante su adecuada utilización, puede realizarse una gestión eficiente de los activos de la empresa, representados por su valor económico. En las Pymes, recaen en la necesidad de implementar sistemas de información contable de tal forma que sea mucho más fácil la toma de decisiones (García, Marín y Martínez., 2006). La contabilidad deriva información indispensable para la administración y el desarrollo del sistema económico. Esta información es proporcionada a quienes toman decisiones, como administradores, propietarios, inversionistas, proveedores, entre otros.

En un ambiente de globalización, los sistemas contables son un concepto fundamental dentro de los procesos de competitividad empresarial, por ello cada vez nacen más estudios teóricos y empíricos que profundizan sus características (Castillo y Villareal, 2014). Una de las estrategias empresariales comunes utilizadas por la empresa, son los indicadores de rentabilidad, lucratividad o rendimiento que pretenden medir la efectividad con que la administración controla los costos y gastos operacionales para convertir las ventas en utilidades (Ortiz, 2004). La información que generan los costos dentro de la empresa, es de vital importancia para llevar a cabo una contabilidad de gestión en el sistema de información contable, porque de forma conjunta establecen su importancia para el establecimiento de la estrategia y desarrollo de las ventajas competitivas, su incidencia en la rentabilidad de las empresas y en la toma de decisiones (Buelvas y Mejía, 2015).

La información se manifiesta como un factor de incidencia para las organizaciones en la concepción y persistencia de ventajas competitivas. Sin embargo, en últimas fechas se ha vuelto indispensable que la información esté bien administrada y sea de buena calidad (Medina y Aguilar, 2013). La información producto de los sistemas de información son de calidad cuando se ha generado en los tiempos oportunos, utilizando las normas contables en su registro; esta información de calidad permitirá a las empresas tomar decisiones que mejoren y aumenten la productividad, así como también hacer planeación financiera y tributaria (Castillo, 2015). 


\section{http://revistainvestigacionacademicasinfrontera.com}

Barrios (2017), menciona que la información que surge del sistema de información contable en la generalidad se los conoce como informes contables, y atento a lo expresado en el párrafo precedente podemos establecer que el Sistema de Información Contable a diferencia de la Contabilidad Clásica es que a través de la Teoría de los sistemas la Contabilidad o Sistema de información Contable ha ocupado dentro de las organizaciones su verdadero papel, el de un instrumental o herramienta muy importante del cual se desprende información que conjuntamente con la producida por otros sistemas y va a permitir que la acción administrativa desplegada a través del Sistema de Administración logre el adecuado uso de los recursos organizacionales. Desde ya con esta nueva visión y misión de la contabilidad en las organizaciones puede colaborar más intensamente en el logro de los objetivos organizacionales participando dentro de sus posibilidades en la toma de decisiones.

\section{Metodología}

\subsection{Diseño de la investigación}

Este estudio se basa en una investigación de campo bajo un enfoque metodológico de corte transversal con el objetivo de determinar con base en las opiniones de los empresarios Pymes del sector industrial de la ciudad de Hermosillo, Sonora, México cual es el grado de influencia que tienen la administración y la calidad de la información de los SIC que usan las Pymes, para tomar más y mejores decisiones y hacer un mejor uso/utilidad de estos sistemas por parte de sus usuarios.

\subsection{Elección y tipo de muestra}


El universo seleccionado para esta investigación se eligió bajo el mismo criterio de estratificación de las empresas utilizado por el INEGI para los censos económicos 2016 de México que fueron obtenidas en noviembre del 2017 y acordados por los integrantes de la investigación, al cual se le anexarán otros criterios tales como: Pymes del sector industrial afiliados a CANACINTRA de la ciudad de Hermosillo, Sonora, México, con una fuerza laboral entre 10 y 250 trabajadores, una antigüedad mínima de cinco años en la actividad. El universo de la población son 796 empresas de las cuales 538 corresponden Pymes con las características elegidas para este estudio. El total de la muestra es de 108 empresas, la cual se fue elegida con la fórmula para una población finita y un muestreo sin reemplazo siguiente:

$$
\begin{gathered}
\mathrm{n}=\frac{\mathrm{NZ}^{2} \mathrm{p}^{*} \mathrm{q}}{\left(\mathrm{Z}^{2} \mathrm{p}^{*} \mathrm{q}\right)+\left[\mathrm{d}^{2}(\mathrm{~N}-1)\right]} \\
\mathbf{n}=\mathbf{1 0 8 . 2 7 \approx \mathbf { 1 0 8 } \text { empresas }}
\end{gathered}
$$

\subsection{Instrumento de recolección de datos}

Para poder realizar la comparación de las respuestas y por ende su categorización y generalización, se utilizará la entrevista abierta apoyada por un cuestionario estructurado donde se analizan tres variables: Variable 1; El Sistema Contable en las Pymes; relacionada con el uso y contenido de un sistema contable en Pymes y Variable 2; Influencia de la información generada de los sistemas contables de las Pymes, relacionada con la calidad de información que genera un sistema contable para la empresa.

Para estimar la confiabilidad del instrumento aplicado, se hizo uso del programa IBM SPSS (Paquete Estadístico para las Ciencias Sociales, por sus siglas en inglés), para obtener el Alfa de 


\section{http://revistainvestigacionacademicasinfrontera.com}

Cronbach en cada una de los items, obteniendo en lo general un coeficiente de .900 . Posteriormente, con la finalidad de detectar errores de diseño y la factibilidad del instrumento, se llevó a cabo una prueba piloto con un mínimo de empresarios con las características de la muestra elegida, para de esta manera realizar los ajustes necesarios en las mismas y así aunada a la prueba de fiabilidad realizada a través del programa SPSS alcancen un elevado grado de confiabilidad. Las encuestas fueron aplicadas directamente a los responsables de las empresas seleccionadas, que permita obtener información que sea verídica y confiable. La información recopilada, organizada y ordenada, será capturada en el programa estadístico SPSS (Statistical Package for the Social Sciences), para su valoración e interpretación.

\section{Presentación, análisis e interpretación de resultados}

Se presentan a continuación los avances más relevantes basados en el objetivo central de esta investigación:

\subsection{Variable 1; El Sistema Contable en las Pymes}

Imagen 1: El sistema de información contable integra información presupuestaria y financiera que influye en la toma de decisiones. 


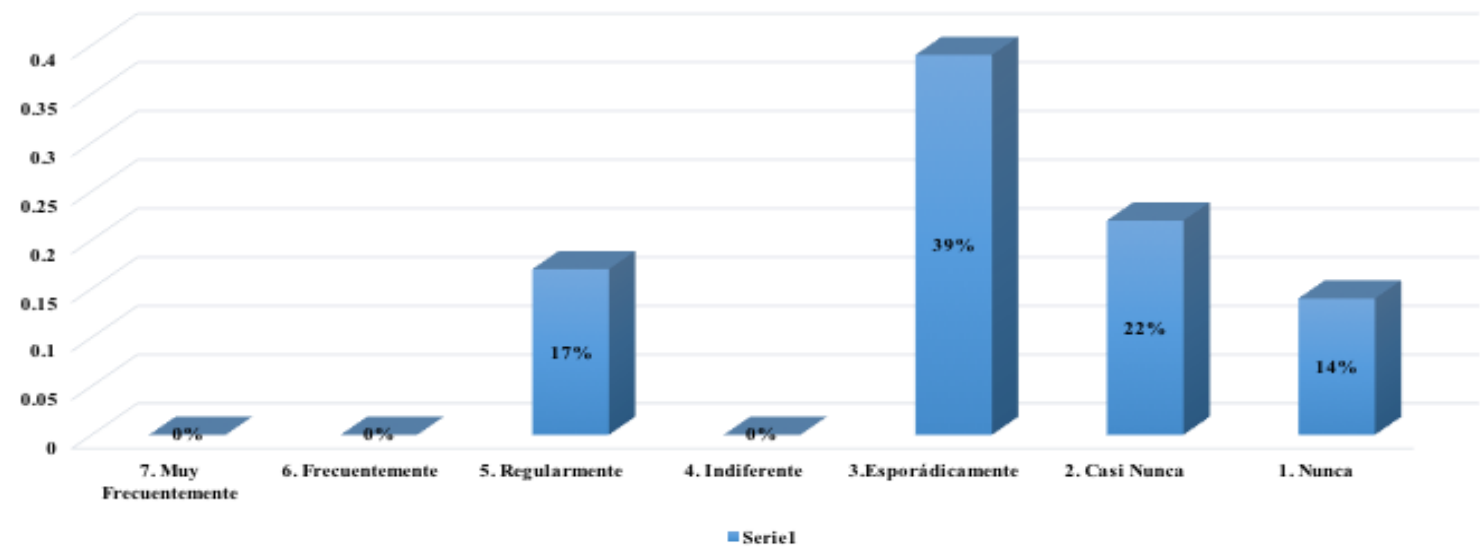

Fuente: Elaboración propia con base en los resultados obtenidos.

La Imagen 1; muestra que de acuerdo al 39\% de las opiniones de los empresarios, esporádicamente las Pymes cuentan con un sistema de información contable que integra información presupuestaria y financiera que influye en la toma de decisiones, el $22 \%$ menciona que casi nunca cuenta con ello, el 17\% menciona que regularmente y el 14\% nunca. Los resultados muestran que, de acuerdo a las opiniones de los empresarios de la muestra analizada, al parecer el empresario Pyme no cuenta oportunamente con información contable que le ayude a tomar decisiones al momento de realizar estrategias presupuestaria y financiera.

Imagen 2: En forma oportuna, el sistema contable genera información susceptible de control y revisión que influye en el ámbito interno y externo de la empresa

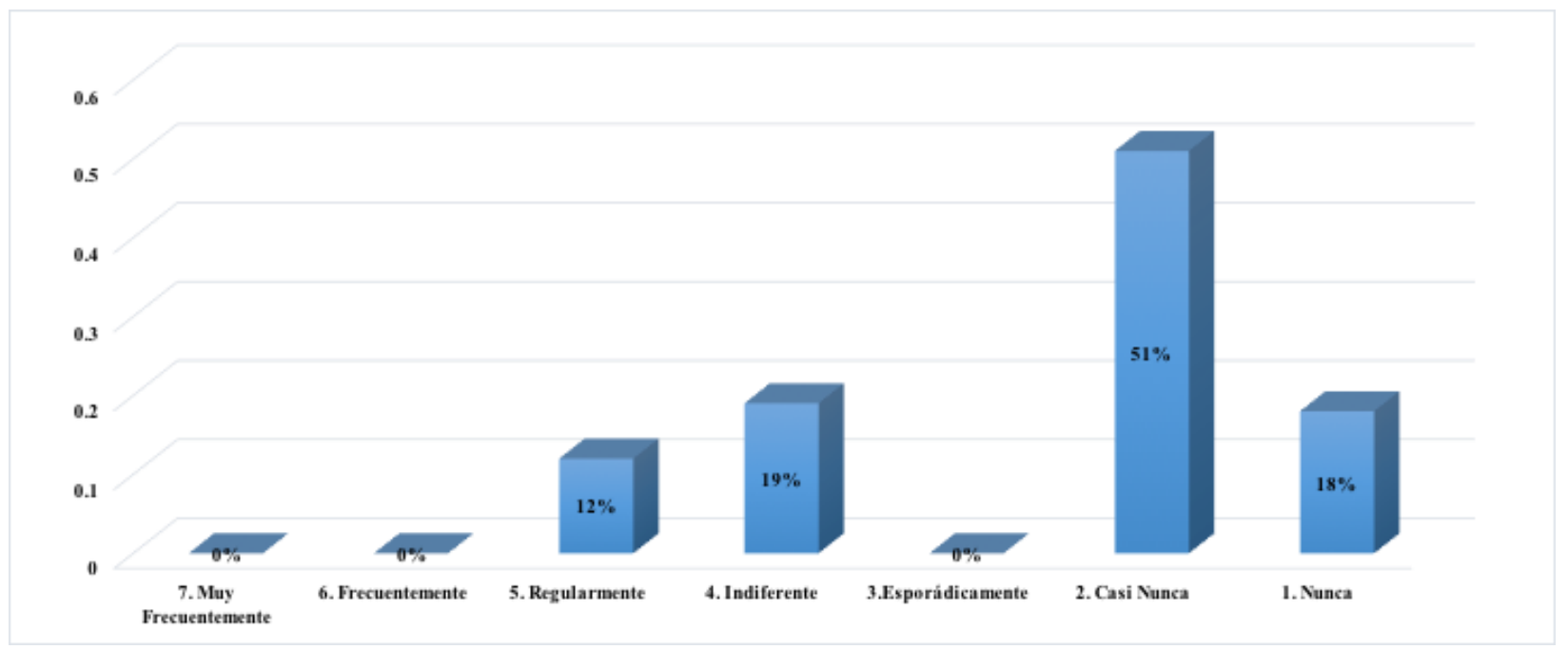

Fuente: Elaboración propia con base en los resultados obtenidos. 


\section{http://revistainvestigacionacademicasinfrontera.com}

La Imagen 2; muestra que de acuerdo al $51 \%$ de las opiniones de los empresarios de la muestra analizada, el sistema contable casi nunca genera información susceptible de control y revisión que sirva como influencia en el ámbito interno y externo de sus negocios, el 19\% no responde, el $18 \%$ opina que nunca y el $12 \%$ opina que regularmente. Los resultados muestran que, al no contar con información el empresario tiene problemas para controlar y revisar los factores que afectan o benefician a la empresa en el ámbito interno y externo de sus negocios.

Imagen 3: La información del sistema contable generada por la empresa reúne las características de transparencia y confiabilidad para la toma de decisiones

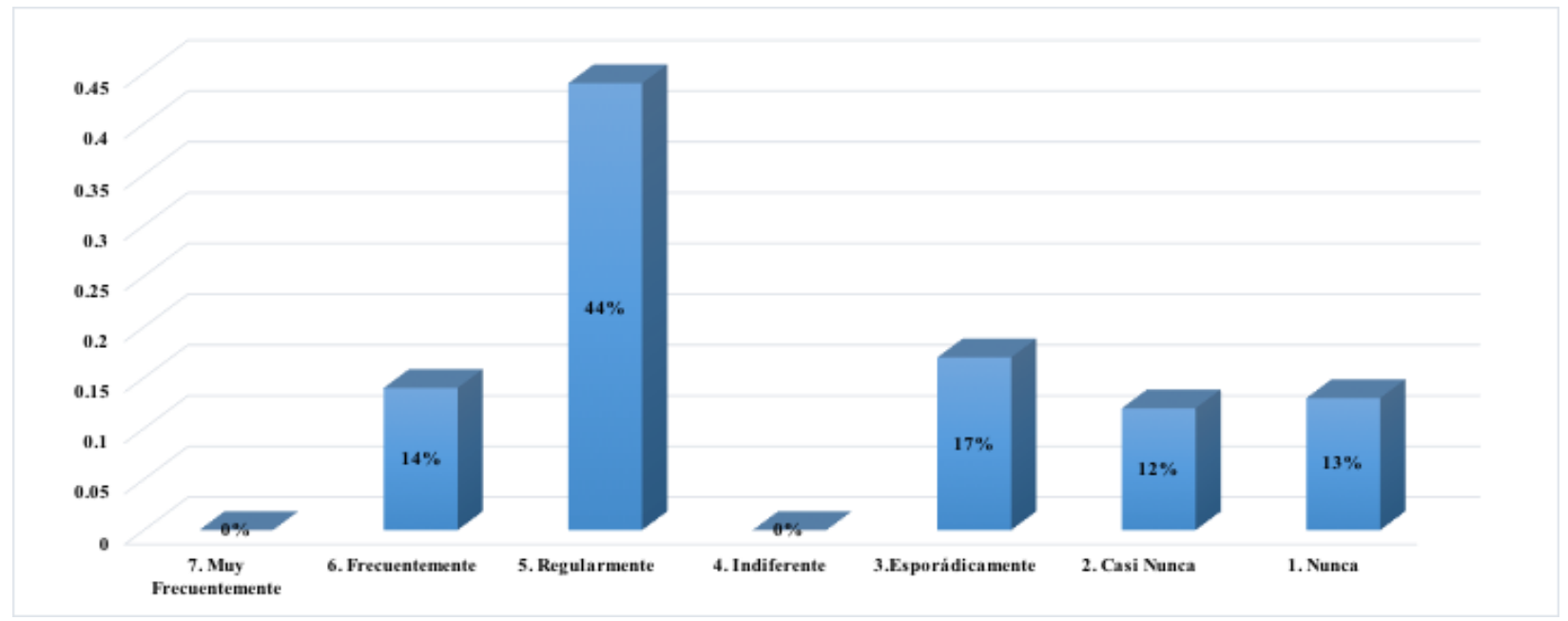

Fuente: Elaboración propia con base en los resultados obtenidos.

La Imagen 3; muestra que de acuerdo al 44\% de las opiniones de los empresarios Pymes, la información contable generada por la empresa, regularmente reúne las características de transparencia y confiabilidad que permite tomar mejores decisiones, el 17\% opina que esporádicamente, el 14\% opina que frecuentemente, el $13 \%$ opina que nunca y el $12 \%$ casi 
nunca. Los resultados muestran que, de acuerdo a las opiniones de los expertos, al parecer el empresario Pyme no tiene confianza plena en la transparencia y confiabilidad de la información durante el proceso en el que se genera lo cual le dificulta la toma de decisiones.

Imagen 4: El sistema contable está creado formalmente con base en las necesidades de la empresa de tal forma que su uso es confiable

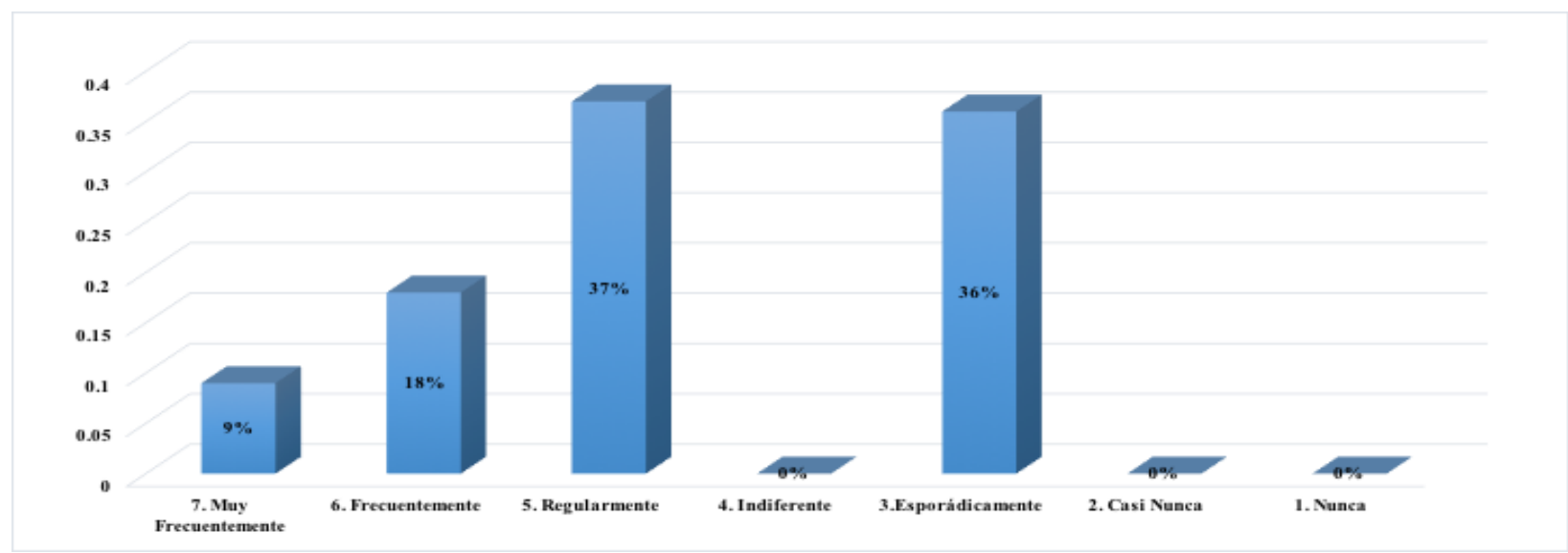

Fuente: Elaboración propia con base en los resultados obtenidos.

La Imagen 4; muestra que de acuerdo a las opiniones del $37 \%$ de los empresarios de la muestra analizada, regularmente el sistema está creado formalmente con base en las necesidades de la empresa de tal forma que su uso es confiable, el 36\% menciona que esporádicamente está formalmente diseñado, el $18 \%$ menciona que es frecuentemente y el $9 \%$ muy frecuentemente. Los resultados muestran que, de acuerdo a las opiniones de los expertos, al parecer el empresario Pyme cuenta con un sistema de información contable regularmente diseñado con base en los objetivos y necesidades de la empresa que le permite tomar decisiones confiables.

\subsection{Variable 2; Influencia de la información generada de los sistemas contables de las Pymes}

Imagen 5. La información financiera permite elaborar estrategias empresariales 
Núm. 28

ISSN: 2007-8870

\section{http://revistainvestigacionacademicasinfrontera.com}

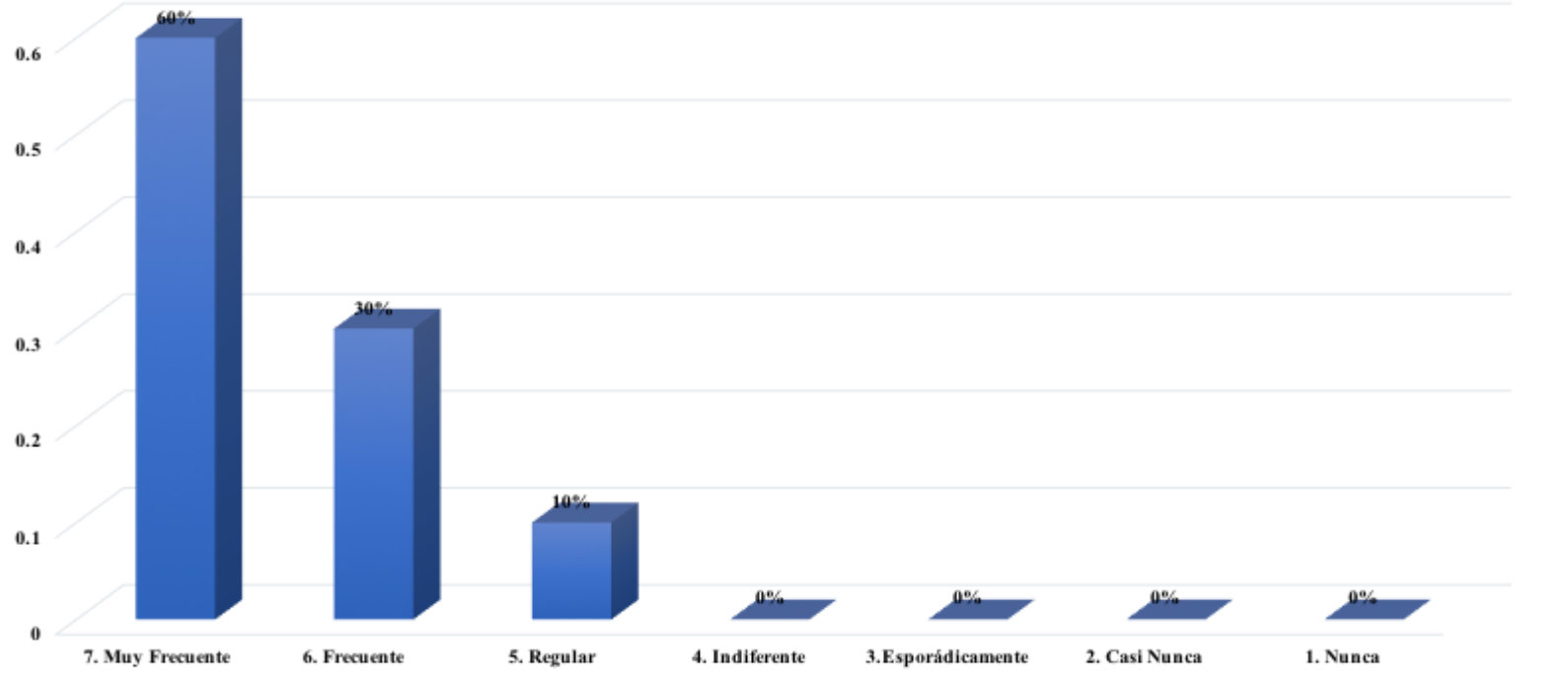

Fuente: Elaboración propia con base en los resultados obtenidos.

La Imagen 5, muestra que para el $60 \%$ opina que es muy frecuente que la información financiera permita a la empresa elaborar estrategias empresariales, el 30\% opina que frecuentemente y el $10 \%$ regularmente. Lo anterior pone de manifiesto que la importancia de la información financiera en la elaboración de estrategias empresariales.

Imagen 6. La información oportuna de un sistema contable ayuda a la realización de un buen análisis financiero que ofrece ventaja competitiva a la empresa 


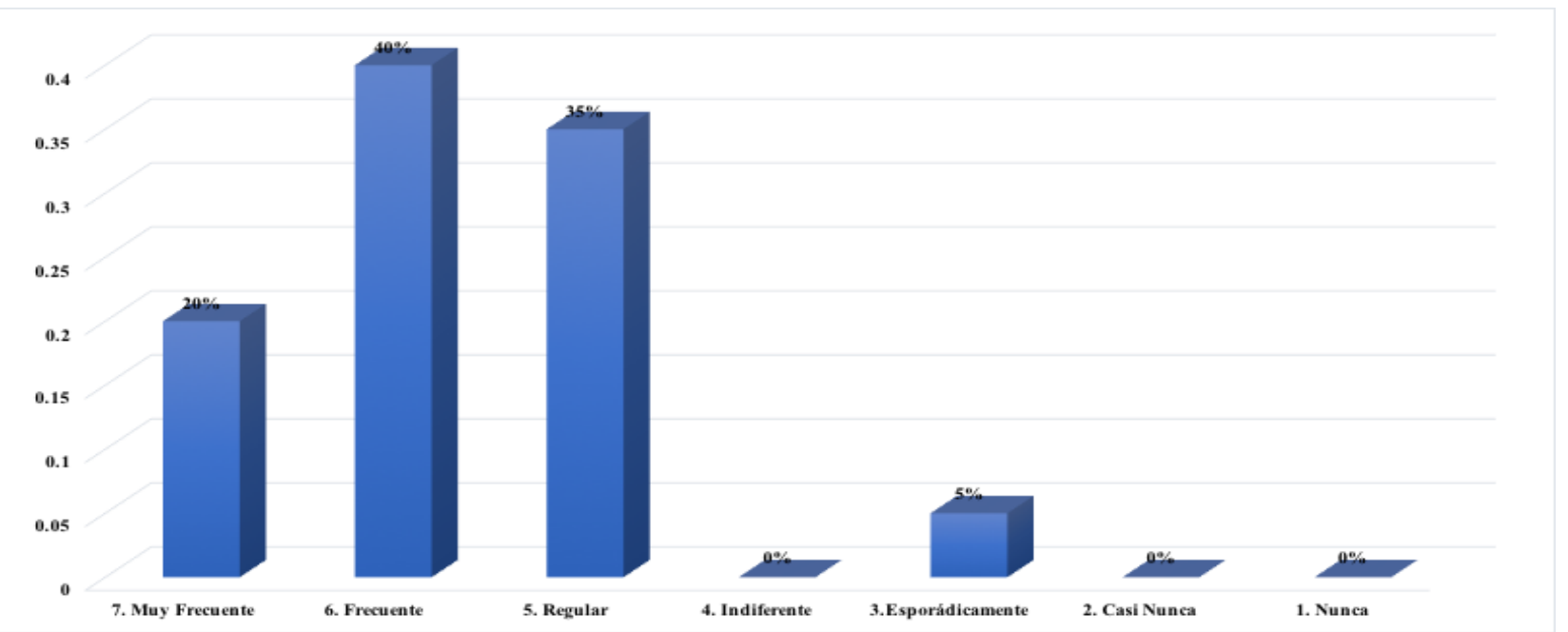

Fuente: Elaboración propia con base en los resultados obtenidos.

La Imagen 6, muestra que el $40 \%$ opina que es frecuente que la información oportuna de un sistema contable ayuda a la realización de un buen análisis financiero que ofrece ventaja competitiva a la empresa, el $35 \%$ opina que regularmente, el $20 \%$ es muy frecuente y el $5 \%$ esporádicamente. Lo anterior pone de manifiesto que al parecer el empresario Pyme se basa en que un buen análisis financiero permite crear estrategias empresariales que le ayudan a lograr ventaja competitiva.

Imagen 7. La empresa utiliza los estados financieros contenidos en los sistemas contables como herramienta para la toma de decisiones.

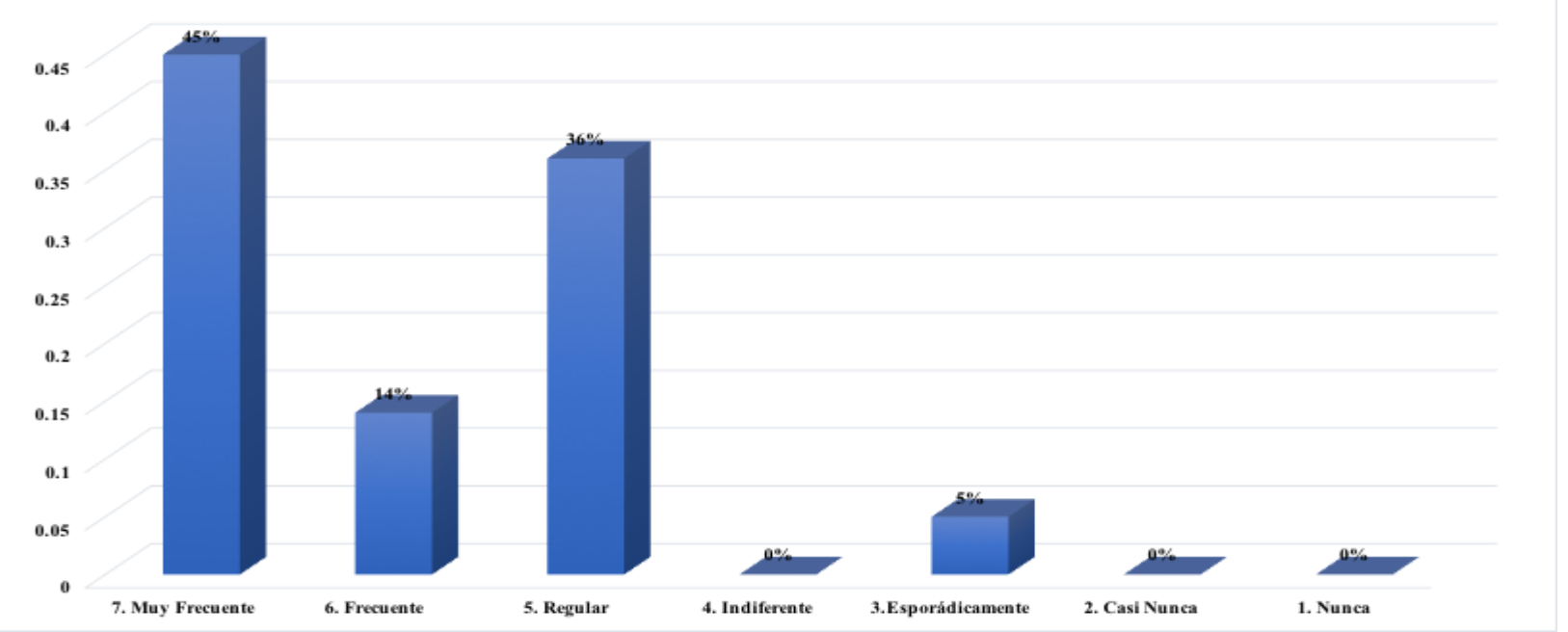

Fuente: Elaboración propia con base en los resultados obtenidos. 
Núm. 28

ISSN: 2007-8870

\section{http://revistainvestigacionacademicasinfrontera.com}

En la Imagen 7, se puede observar que el $45 \%$ de las opiniones de los empresarios Pymes muy frecuentemente utiliza los estados financieros contenidos en los sistemas contables como herramienta para la toma de decisiones, el 36\% lo utiliza regularmente, el $14 \%$ lo utiliza frecuentemente y el $5 \%$ esporádicamente. Lo anterior pone de manifiesto la utilidad de los estados financieros en la toma de decisiones.

Imagen 8. Existen mecanismos de control que miden la veracidad de la información suministrada por el sistema de contabilidad general

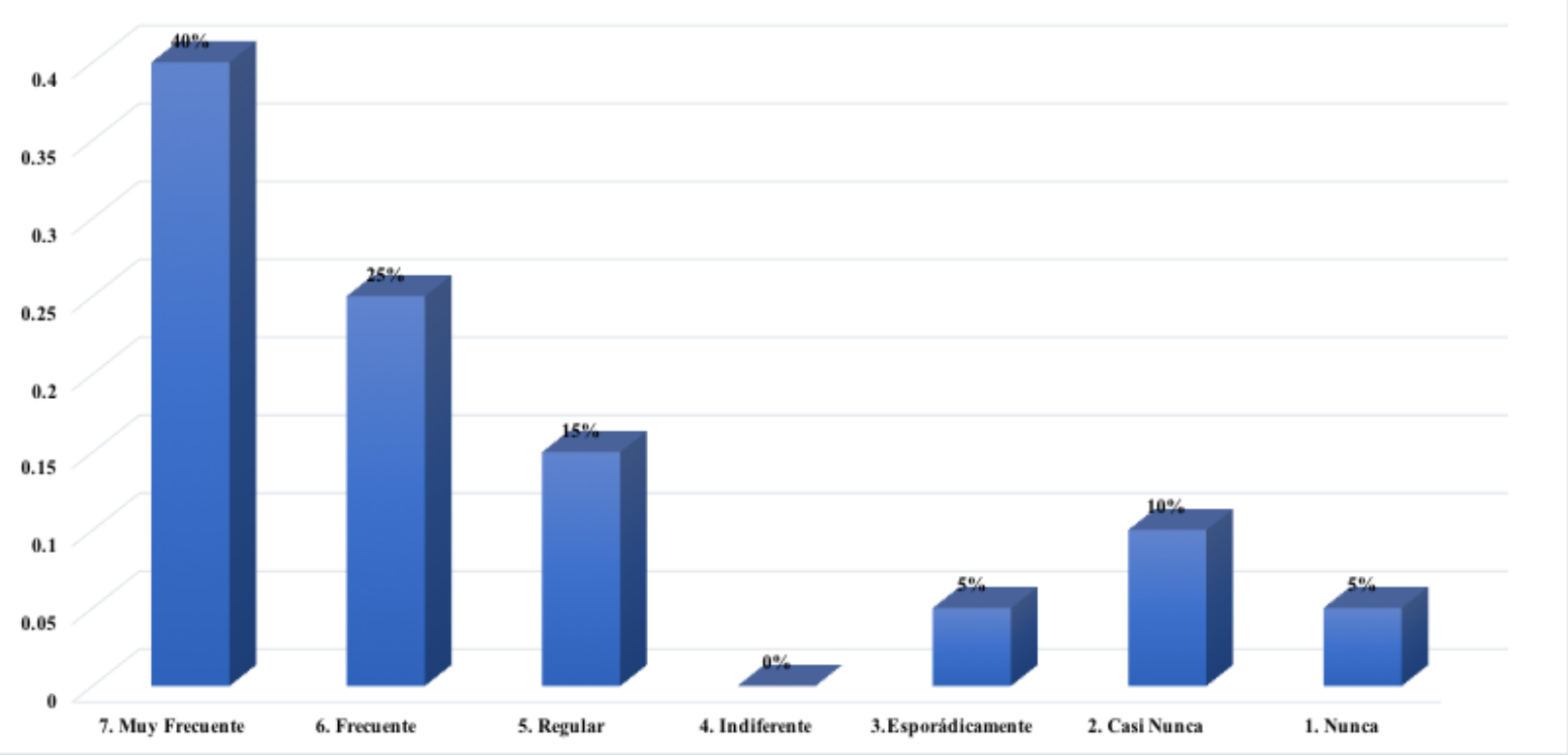

Fuente: Elaboración propia con base en los resultados obtenidos.

La Imagen 8, muestra que de acuerdo al $40 \%$ de las opiniones de los empresarios, muy frecuentemente existen mecanismos de control que miden la veracidad de la información suministrada por el sistema de contabilidad general, el $25 \%$ opina que frecuentemente, el $15 \%$ casi nunca y una igualdad de respuesta del 5\% que existe esporádicamente y nunca. Los resultados ponen de manifiesta que la veracidad de la información depende de la calidad en los sistemas contables. 


\section{Conclusiones e implicaciones sociales y empresariales}

Indudablemente, uno de los más grandes retos que se presentan y que debe superar toda pequeña o mediana empresa, incluso desde antes de iniciar sus operaciones, es el de desarrollar e implementar mecanismos que le permitan administrar, gestionar, almacenar, analizar, consultar, producir y utilizar la información y datos financieros que se generan en la entidad. Derivado de esta necesidad y reto administrativo surgen los sistemas contables, el conjunto de mecanismos y herramientas utilizadas para mantener el control de los recursos y las actividades que realiza una organización, lo cual también ofrece como ventaja ayudar a cumplir de manera puntual y constante con las obligaciones fiscales y contables de la entidad, además de ser de particular importancia para aquellas Pymes con planes de expansión, crecimiento y ventaja competitiva.

Los resultados obtenidos en la presente investigación ponen de manifiesto que dentro de la Variable 1; El Sistema Contable en las Pymes; se muestra como el empresario está consiente de la importancia que tiene el uso y contenido de un sistema contable dentro de la empresa, posiblemente está consciente de que la información oportuna generada por un sistema contable permite lograr una eficiencia en la gestión empresarial, lo cual permite poder controlar a tiempo todos aquellos factores que afectan o benefician a la empresa que busca una ventaja competeitiva. En la Variable 2; Influencia de la información generada de los sistemas contables de las Pymes; se muestra que el empresario está consciente de la importancia que tiene la calidad de la información que genera el sistema de contabilidad en las estimaciones presupuestarias, pero aun así desconoce la calidad que le ofrece la información de los indicadores financieros para evaluar los resultados dentro de su gestión empresarial. Sin embargo, está consciente de que los análisis financieros son la base del trabajo para una planeación estratégica empresarial encaminada a la ventaja competitiva. El empresario es consciente de que la calidad de la información que genera el sistema contable depende el éxito o fracaso de la toma de decisiones. 


\section{http://revistainvestigacionacademicasinfrontera.com}

Las Pymes tienen que abandonar el miedo a la formalización empresarial y que los administradores dejen de considerar a la contabilidad, como un gasto obligatorio y se empiece a ver como un instrumento de apoyo en la búsqueda de la expansión y la excelencia. El empresario tiene que comprender que la información que ofrece la contabilidad es favorable para los objetivos de la empresa, por medio del suministro de información eficiente a la gestión puede tomar sus decisiones con base a información que se ha preparado y presentado pensando en satisfacer las necesidades de información principalmente de los usuarios internos de la empresa; ya que generalmente en las Pymes, sobre todo en las de menor tamaño, no hay usuarios externos interesados en obtener información a la medida de sus necesidades, con excepción del Estado, que siempre será un usuario importante de la información generada por todos los tipos de empresas.

A modo de conclusión final y tomando en cuenta los planteamientos anteriores, cabe resaltar que la administración de la información no incide para la toma de más y mejores decisiones, se requiere profundizar en la relación al ser estos hallazgos el resultado de una investigación en un grupo de Pymes, por lo que no se pueden generalizar a todas las organizaciones de este tipo. Por otro lado, la percepción de la influencia y calidad de la información de los sistemas contables en la toma de decisiones aquí planteados, debe extenderse a otros puntos no analizados, tales como las características comparativas de cada uno de los factores de éxito o fracaso que otorgan los sistemas de información contable al momento de la toma de decisiones por parte de las Pymes en razón a su giro económico. 
Estos resultados comprueban el objetivo planteado en la investigación donde se hace ver desde la perspectiva del empresario Pyme, el grado de influencia que tienen la administración y la calidad de la información de los sistemas contables que usan las Pymes, para tomar más y mejores decisiones y hacer un mejor uso/utilidad de estos sistemas contables por parte de sus usuarios de tal forma que de ventaja competitiva a la empresa.

La realización de este proyecto se considera viable ya que Sonora, cuentan con un sector económico muy dinámico en sus diferentes sectores, tanto el industrial, el comercial y el de servicios. Para Sonora, la Pyme ocupa un lugar preponderante en cada uno de ellos. En esta investigación se pretende demostrar que existe una alta necesidad de realizar más trabajos de investigación relacionados con el tema, con enfoques específicos por sector, región y actividad, así como darlos a conocer a las cámaras de las empresas y a las asociaciones de profesionistas para que difundan los contenidos entre los empresarios Pymes.

De la misma manera es necesario dejar claro que la metodología aplicada en este estudio podrá ser utilizada y ampliada en el proceso de la misma investigación y en otros casos. Los avances de resultados de este trabajo servirán para orientar a las Pymes hacia la reflexión sobre la importancia de la aplicación del conocimiento para la elaboración de planes efectivos para la capacitación de estrategias encaminadas al uso y aprovechamiento de la información generada en los sistemas contables. También serán útiles para un estudio cuantitativo posterior más amplio, el cual abarcará más empresas locales y permitirá obtener información sobre otras poblaciones en el estado o nacionales.

\section{Referencias bibliográficas}

Barrios, E. A. (2017). La contabilidad y los sistemas de información contable en las organizaciones. Ediciones UNPAedita, Argentina. 


\section{http://revistainvestigacionacademicasinfrontera.com}

Buelvas-Meza, C.G. y Mejía-Alfaro, G.E. (2015). El papel de la contabilidad de gestión en el sistema de información contable y su incidencia en la rentabilidad de las empresas. Revista panorama económico, Vol. 22, pp. 91-108.

Castillo Pérez, A.A. (2015). Importancia de la calidad de la información de los sistemas informáticos contables en las empresas. In Crescendo. Ciencias Contables y Administrativas, 2(2), pp. 123-141

Castillo, C.; Villareal, J. (2014). Los Sistemas de Información Contable y el Balanced Scorecard en las pymes industriales de Pasto.

García, D., Marín, S., y Martínez, F. (2006): La contabilidad de costos y rentabilidad en la PYME. Contaduría y Administración, México, abril-junio, no. 218, pp. 39-50.

Hernández-Chaustre, S. A. (2006). La pyme en Latinoamérica. En Sergio Alberto HernándezChaustre; Sergio Calderón-Acevedo; Jorge Humberto Botero; Enrique Barriga; Margoth García; Juan Manuel Lesmes; Mauricio Jaramillo; Gustavo Aristizábal; Gustavo Ardila; Camilo Téllez; Claudia Vega; Marcial del Castillo; Natalia Jiménez \& Maribeth Murphy. El gran libro de las pymes - Información Financiera. Bogotá: Casa Editorial El Tiempo. 22-27.

Hitt, Michael A.; Hoskisson, Robert E. \& Ire- land, R. Duane (2001). Strategic manage- ment. Competitiveness and globalizations. New York: Thomson South-Western College Publishing.

Horngren, Ch., Foster, G. y Datar, S. (2002). Contabilidad de Costos: Un En \pm oque Gerencial. Décima Edición, Pearson Educación, México

Johnson, Gerry; Scholes, Kevan y Whittington, Richard (2007). Dirección estratégica. 7a ed. Madrid: Prentice Hall. 
Medina Quintero, J. M. y Aguilar Gámez, P. E. (2013). Administración y calidad de la información de los sistemas de información contable de las Pymes. Cuadernos de Administración, Universidad del Valle, Vol. 29, No. 49, pp. 8-16.

Peña, A. E. (2011). Eficacia del sistema de información contable de las Pyme merideñas calificadas en el régimen ZOLCCYT Visión Gerencial, núm. 1, enero-junio, 2011, pp. 95107

Rueda Delgado, G. y Arias Bello, M. L. (2009). Los sistemas de información contable en la administración estratégica organizacional. Cuaderno de Contabilidad, 10 (27), 247- 268.

Schekaiban, N., \& Ripoll Feliu, V. M. (2005). Estudio comparativo sobre investigación en contabilidad de gestión. España-México. Anais do Congresso Internacional de Custos, Florianópolis, SC, Brasil, 9.

Vaivio, J., y Sirén, A. (2010). Insights into method triangulation and "paradigms" in interpretive management accounting research. Management Accounting Research, 21(2),130-141.

\footnotetext{
Para citar este artículo

PATRICIA AGUILAR TALAMANTE/ALMA BRENDA LEYVA CARRERAS/JOSÉ ALFREDO HEREDIA BUSTAMANTE/MARÍA CANDELARIA GONZÁLEZ GONZÁLEZ. PRINCIPALES PROBLEMAS DE LA PYMES POR FALTA DE INFORMACIÓN OPORTUNA GENERADA EN SUS SISTEMAS CONTABLE. RIASF. NÚM. 28, JULIO-DICIEMBRE (2018), ISSN 2007-8870. PP. XX-XX.
} 